\title{
Intraductal Papillomatosis
}

National Cancer Institute

\section{Source}

National Cancer Institute. Intraductal Papillomatosis. NCI Thesaurus. Code C7363.

A neoplastic process characterized by the presence of multiple intraductal papillomas. 